draguer les dépóts limoneux par aspiration, à la manière des dragues suceuses des ports maritimes; mais, au lieu d'employer une énergie étrangère pour produire l'aspiration, M. Jandin a utilisé celle de la chute créée par le barrage de la Djidiouia.

Lorsque les dépôts sont peu consistants, l'aspiration naturelle suffit pour provoquer leur entrainement $(20$ à 25 pour 100 de vase molle, pour 75 a 80 d'eau). Pour les dépôts ayant acquis une certaine consistance, M. Jandin a disposé, à l'entrée du tuyau, une petite turbine qui entraine une scie circulaire hémisphérique qui découpe des copeaux dans la vase; ceux-ci sont entrainés dans le tuyau d'évacuation par le courant d'eau, et rejelés en dehor's (dans la proportion de 10 à 15 pour cent pour les dépòts récents de 1 à 3 ans de date, mais pouvant tomber à 6 et même à 4 pour les vieux dépôts). Le draguage complet s'est effectué jusqu'à $300 \mathrm{~m}$. du barrage; la conduite flottante d'évacuation comportait des joints flexibles, et était soutenue par des flotteurs.

Pour entretenir le dévasemenl, M. Jandin a préconisé l'emploi de tubes suceur's, de petit diamètre. répartis en divers endruits, et reliés à la conduite grénérale d'évacuation. C'est à notre avis une excellente solution.

En général, le ou les usagers de l'eau emmagasinée dans un réservoir artificiel, doivent restituer à l'aval une certaine quantité d'eau fixée par les règlements. Lorsque cette quantité d'eau n'est pas trop faible, et que l'apport de la rivière n'est pas trop grand, on peut évacuer les dépôts d'une manière continue, en disposant l'une des priies d'eau correspondantes assez près du fond, au moyen d'un petit siphon par exemple. Le courant d'eau continu qui se forme devant cet orifice entraine les dépôts au fur et à mesure qu'ils viennent se former dans son voisinage. Ce dispositil est actuellement employé au barrage du Gouffre d'Enfer; toutefois, pour qu'il soit réellementelficace, il faudra disposer plusieurs prises en différents endroits, de manière à réaliser les suceurs de M. Jandin.

Nous avons dit plus haut, à propos des évacuateurs de crues, que l'on pouvait, dans certains cas, être conduit à employer des siphons s'amorçant automatiquement, et débitant sous la hauteur totale de la retenue. Au lieu de faire déboucher ces siphons presque au niveau de leur point le plus élevé, on peut les faire déboucher près du fond, de la sorte, les clues qui amènent les dépóts se chargent elles-mèmes de les évacuel en partie. Dans ce cas. il est bon, pour s'éviter toute surprise en cas d'obstruction intempestive de l'orifice d'entrée du bas du siphon, de ménager un second orifice vers la partie supérieure, cet orifice, obturé en temps ordinaire, ne servant que d'orifice de secours. D'autre part, le siphon peut se terminer, à sa partie inférieure amont, par plusieurs orifices disposés en des endroits différents, de manière à opérer successivement des chasses en divers points,

Pendant longtemps, le barrage en maçonnerie a été considéré comme quelque chose de mystérieux, qu'un ne devait aborder qu'avec beaucoup de prudence. Nous espérons que nos articles, un peu longs peut-être au gré du lecteur, montreront qu'il est possible de construire des murs réalisant le minimum de maçonnerie, tout en possédant une stabilité qu'on ne saurait mettre en doute.

\section{H. BeLlet.}

\section{Le nouveau Projet de transport à Paris de l'Énergie du Rhône"}

La nouvęlle solution consiste dans la creation d'un barrage unique, de sgrande hauteur, à la sortie de la partie la plus étroite des gorges du Rhone, de façon à nover celles-ci sur toute leur longueur, et ramener ainsi le plan d'eau supéricur du fleuve au niveau qu'il devait avoir autrefois avant la corrosion de son lit. Les points qui paraissent le plus propice pour l'édification de ce grand banrage se trouvent au voisinage de la stadion de Génissiat (Ain), soit en amont du ravin de la Dent, soit en amont de Monthoux, soit encore un peu plus a l'aval ou id l'amont, si la suite des éludes en démontie lopportunité.

hans le précédent projet, on avait adoplé la cote 330,50 pour le nivcau de l'eau à la frontiere suisse, et celle de 262,25 pour l'evacuation des turbines a Nonthoux. La différence de niveau de $68,25 \mathrm{~m}$. dulut réduite a 63 mìtaes environ jon la perle de charge, et par la pente nécessaire a l'écoulement de l'cau principalement dans les tunnels.

En rupportant cos nivellements au nivellement général de la France, la cote d'eau qu'il est permis dattcindre il la frontiere suisse serait environ $332^{m}, 50$, au lieu de 330,50 ; clle serait voisine de 262,50 dans la gorge de Génissiat ; dou une dilférence de nivaru d'environ 70 metres (1).

Dans la nouvelle solution, qui ne comporte plus de tunnels, grace a la section offerte au passage de l'eau, l'écoulement sera considérable; l'écartement des roches qui limiteront le lit du fleuve varie de 100 is 150 mètres, et meme en ccrlains points ir 200 mètres, et une pente totale inféricure it $0^{\mathrm{m}}, 50$ suffira ì assurer le débit nécessaire jusqu'à Monthoux; le niveau du fleuve sera en ce point it la cole 332 mètres.

Jusqqu'ì Grésin, le résultat serait le même que dans l'ancien projet; le Rhone s'écoulcrait suivont une nappe de 8 mètres audessus de la plate-forme rocheuse siluée en cet endroit, où le lit aurait environ 60 metres de largeur. Il s'elèverait au bas de Bellegarde de façon i envahir le bas de la pilate-forme qui forme un promontoire au confluent du thone et de la Valserine, et remonterait le cours de cet aflluent jusquau nivcau des vannes de décharge de la première usine située sur ce torrent. Un petit lac se formerait en cet endroit, mais il n'y aurait que quelques maisons i expropirier au bas de Bellcgarde, et tout le reste de la ville n'aurait rien à en souffrir ; naturellnment, l'usine de Bellegardo serait rachetée, et l'énergie serait rendue aux usines existantes dans celle localité. A l'aval de Bellegarde, les gorges sont, en général, très encaissées, avec des palois presque verticales, et il n'y aurait que lo hameau d'Essertoux qui devrait aussi etre exproprié.

Ainsi, dans l'onsomble, le projet ne présente pas de difflcullés au point de vue de la cole supérieure des eaux, meme à 332,50 . Il cxiste dans d'autres pays, notamment en Allemagne, au récent barrage de la vallée de l'Urft, des exemples de vallées submergées arlificiellement sur des étendues bien plus considérables en vue de réaliser un transport d'énorgic hydro-électrique (2). Cepenclant, il s'agissait, pour celte entreprise, dune puissance constante de 3.000 kilowalts, environ, alors qu'un ouvrage analogue en oréera une ici 50 fois plus considérable.

D'ailleur's, les auteurs du projet sont tout prets, si l'administralion ou le Parlement le désire, à abaisser la cote du plan d'eau supélieur à 327 mètres seulement, ce qui dénoierait quelques maisons aicluellement compromises ì Bellegarde, et même toute la plaine de Collogny dont on n'a plus besoin pour crêer une réserve d'eau. Il est bien évident, en effet, que le cube emmagasiné utilement dans le barrage unique, meme a la cote 327 , sera plus de dix fois supérieur à cclui que l'on avait primitivement cherché à obtenir.

Ni la route de Bellegarde à Geneve, ni meme la ligne de chemin de fer, ne seraient compromises par la retenue.

La vitesse du courant serait tellement réduite dans la retenue $\left(0^{\mathrm{m}}, 05\right.$, par seconde environ en basses eaux $)$, qu'il n'y aura pas à redouter les effets de l'érosion de la roche ni meme des terres des rives.

()) Pour le premier projet, voir la Houille Blanche, no de janvier 107. (1) Le 24 septembre 1906 , l'eau etait à la cote 332,03 à la frontière et a 262,30 vers le ravin de la Dent.

(2) Voir La Houille Blanche, nº de juin $\$ 906$. 
Les quelques ponts rendus inutilisables seraient remplacés par c'aubres construits alt-lessus du niveat superieur de la reteme ; la passeralle de Génissiat serait remplacée par le chemin couronmant le bstrrage. Tous ces travaux ne présentent aucune difficulté, car l'on peut toujours installer les ponts ou passcrelles nécessaires avec des portées de 80 à 100 mètres, en des points où des rétrécissements locaux s'y prêtent.

Barrage. - Grace aux falaises de Génissiat, d'un calcaire très résistant, et au caractêre rocheux du fond du lit, l'exécution du harragie ne parait pas devoir présenter de difficultés malgré sa hauteur excoptionnelle de 78 mètres au mininum. Il est facile, du reste, de lui donner une soliclité exceptionnelle, grace aux circonstances loxales. Il aura en plan la forme d'une voute is convexilé loumere ver's l'amont, et s'appuyant sur les falaises des rives servant de culées. En outre, le barrage n'aura pas ì servir de déversoir, comme le barrage de Grésin du premier mémoire ; en eliet, on trouve, sur la rive droile, au sonmet de la falaise alcaire qui sora dérasée à cel eflet sur une certaine largeur, tout lespace nécessuire pour y insladlet un canal de décharge.

L'étiaro all point choisi vers Gromissiat ètant in la cote 262,50, la hauteur de la retenue sera de $332-262,50=69^{\mathrm{m}}, 50$; la hauteur intale du barrasse depuis le fond du lit, sans compter ni les fondafions ni la revanche au-dessus de la relenue, sera d'environ 3 mètres plus grande, soit $72^{\mathrm{m}}, 50$. Cette hautcur est exceptionnelle, mais les circonstances locales très favorables permettent de considórer l'ouvrage comme parfaitement réalisable, et devant offrir une grande solidité, grace a la possibilité de lui donner une forme cintréce en plan vars l'amont. et de l'encastrer solidement dans les falaises des deux rives (1).

La scule difficulté d'execution sera elle de la fondation, qui devra stro faite avec les précautions les plus grancles adoptés pour ce genre de travail, cest-i-dire sur le rocher mis it nu, et iuprès elude de celui-ci par de nombreux sondarges préalables. On conmencera par construine, it l'amont de l'emplecement du barrase définitif, un harrage provisoire it piorres perdues, conslilus comme celui de la premiere solution et arasé à la même hauleur, qui permettra de dévier les eaux du Rhone, jusqu'aux crues ordinaires de 600 à 650 mètres cubes, par des gaheries nerobes dans la rive droite el debouchant it l'aval du grand barrage. On élanchenta ce barrigse provisoire par une tôle verticale noyéc dans sal masse, de façom à obtenir à l'aval un calme suffisant pour pouvoir fonder, à lamont ef it laval de la fondation projetée, deux murs élanches de 8 mètres d'épaisseur s'élevant à (un,50 all-dessus de létiage. Ce travail sexeculera à l'air comprimé, au moyen de caissons mobiles, par le sTsteme dejà employe aux ports de Gones, de la Palisse et de Marseille ; les vides licissés entre deux blors consécutifs conshlruits dans les caissons seront mis a sec alu moyen de mureltes en brique et ciment, exćculées sous leau par des scaphandriers et aremplis ensuite de maconncrie.

Entre ces muls, on épuisera et on construira à see le corps de la fondation, puis le barrage scra exécuté entièrement en maconnerie de mellons et mortier de ciment.

Les épaisseurs seront déterminées, à différentes haubeurs, en vérifiant que sur le parement aval le chiffre de pression le plus ćlcvé, soit inférieur à l'effort limite admissible ; à l'amont, nonseulement on évilera tout travail d'extension, mais, suivant la théoric de Maurice Lévy, on réslisern un travail de compression de la maconnerie supérieur ì la pression lyydrostatique de l'eau en pleine retenue, el inféricur, quand la retenue sera vide, aux $3 / 2$ de l'elfort limite admis pour la maçonnerie d'avial.

Tout contact de l'eau avec la maçonnerie proprement dite du barrage sera évilé en construisant ì 2 mètres en amont de celuici un mur de garde de 1 mètro à la cròte et de 3 mètres à la base, s'appuyant contre le barrarge par des voules a axe vertical de 2 metres d'ouverture, et d'une énaisseur tolale aux naissances correspondantes.

La mise en charge du barrage se fera dans une période d'étiage, en rabaltant successivoment sur l'entrée de chacune des trois ga lories de dierivation des masques prćpantés d'arance, et en constiruisant pour chacune, derière le masque qu'on vient de rabattre, un bouchon en maçonneric de 20 métres de longueur traversé par 5 tuyaux en fonte de 1 mètre de diamètre, terminés à l'aval par des robinets-vannes qu'on laissera ouverts. A la premiere clue; on fermera une partie des robinets, de façon à élever pro.

(1) Voir à ce sujet. dans le no de juin 1906 de La Houille Btanche, le nouveau barrage du Groton qui a $92 \mathrm{~m}$. de hauteur, et le barrage loosevelt, qui en a 79,50 . gressivement la retenue tout en laissant dans le Rhône un débit suffisant. Chaque fuy:au peut en elfet évacuer environ 20 mètres cubes sous la charge de la retenue, ce qui fait 300 mètres cubes pour les 15 tuyaux ; ce débit se réduit à 100 mètres cubes poul: une charge de 9 mètres au-diessus de l'étiage. Ces tuyaux scrviront aussi on exploitation de moyens supplementaires pour lévacualion des crues et éventuellement plus tárd de désableurs ; wu bien on metlril des rannes au fond de tours correspondantes, pladés it l'amont des tunnels, ainsi qu'on a procédé au barrage de l'Urft.

Evacuation des crues. - Pour l'evacuation des crues, on étahlira, a l'an'al de l'emplacement choisi, un large deversoir à cascalde dont le seuil serát placé à la ecte 326 ; l'eau y accédera par un canal de $60 \mathrm{~m}$. de longueur sur $8 \mathrm{~m}$. de large; il sera muni de 5 vannes Stoney, permettant de régler le débit d'évacuation, jusqüa concturrence de 1,350 mètres cubes à la seconde, tandis que les plus grandes crues ne dépassent pas 1,150 mètres et que, d'autre parl, les canaux d'alimentation de l'usine peluvent donne 330 métres culses, dóbil donné de meme par les 15 tuyaux des gualeries de dérivation.

On comiluatlra la violonce de l'icaulement au sortir des vanres Je déhurge en Garrgissant lc ravin de la: Dent, et en constituant des échelons dans le lit, ainsi qu'on a fait pour les vannes de décliarge du barrage de l'Urft.

La grande profondeur du canal d'amenée est motivée par les valtiations de niveau de la retenue.

Déversoir. - Liespace compris entre le barrage et les vannes de prise dicall, soil sur 100 mètres cnviron de longueur, scia occupé par un déversoir à bord ondulé, dont la crête ser'a arasée à la wole 332,15 , soit 15 centimètres plus haut que la retenue l'eau déversée s'écoulera dans une chambre dont le fond sera mis en relation avec l'aval au moyen d'une trompe sinueuse, afin de combattre laccólération de l'eau el la violence du jet à la sortie. Si, par suite d'une circonstance extrandinaire, les vannes de décharge neélaient pas soulevées, l'eau qui s'écoulerait an-dessus d'elles (soil 2 meires sur les vames ayant 50 metres de développement), et celle qui serait evacue par le déversoin, avant d'atteindre la cote de couronmment du barrage, soil $1^{\text {m }}, 85^{\prime}$ sur le déversoir dont la crêle rluiveloppée aura 150 mètres, donneront ensemilne un débit de 1,100 mòtres cubes, chiffre supérieur ì celui des crues ardinaires qui sont de 800 metres cubes. Or poilura angmentes le déversoir en le répétant sur un autre point, si on le juge utile

Prise d'eau el canal d'amence. - Les ouvrages de prise d'eau serout, élablis sur le camal ci-dessus servant à l'évacuation des crues ; ils seront formés chacun d'un hassin d'entrée fermé pa! un grillage en cormietres, et de deux vannes Sloney, de $5 \mathrm{~m}$. de Lalmeur sur $8 \mathrm{~m}$. de hantenr. qui. traversées il la vitesse de 1 metre, debiteront chaoune 10 mitres cubes a la seconde.

Dorriare ces vamnes, existera une chambre de prise, avec descente de $2^{\text {mit }}, 70$ de diamitre pour chaque acicoupiement de deux groupes électrogincs. Les vanmes Stoney permettront l'isolement complet de chracun de ces accouplements, la mancuvne individuelle des groupes sera assurée par un distributeur à servomoteur. Les vamnes stoney seronl mues eleclriquement a distance, et prolégées par une conslruction couverte.

Chaque descente sera constiluec nar un tuyau en acier de $2^{\mathrm{m}}, 70$ de diamètre, logé dams un puits incliné, creusé dans la roche, et enrobé dans du béton garnissant les vides.

Les grilles placées à l'entrée des cluambres d'eau seront nettoyécs automatiquement par un moyen mécanique.

Pertes de charge. - En prevoyart $0^{\mathrm{m}}, 50$ de perte de charge, tant dans lo canal damenée, que dans lets grilles et descentes, on sera au-dessus de la vérité, meme en tenant comple de toutes les pertes qui ne peuvent s'évaluer exactement.

La chute disponible, déduite du niveau de la retcnue 332 mètres. et du niveau d'aval 262,50 en déduisant en outre la perte de charge de $0^{\text {m }}, 50$ sera de 69 metres ; mais elle pourma s'abaisser à 67 mètres pendant les hautes equx ou si, pendant les basses eaux, on pratique l'emma:grasinement pendant une partie de la journée, et cruon porte a certaines heures le debit au double de sa moyeno. lans les tris grandes crues, si le Rhone monte de 3 mètres au maximum, ant-clessus de l'étiage, la chute disponible ne serait pas inforieure is 6' mètres.

La puissance motrice hydraulique brute minima, en ne tenamt compte que des débits du Rhone, de l'Arve et autres affluents. atteint pendant 3 mois, $120 \times 69 \times 10=83000$ chevaux, qui pourront 
etre chaque jour doublés ou menie triplés pendant quelques heures par. emmagasinement.

Le débit noyen du Rhơne \&̀ Giénisiat peut s'établir en comptant sur celui de 270.08 mo (fu'il donne à la sortie du lac, augmenté du desbit de l'Arve, très inconstant, et que nous fixerons arbitrairement à 50 mìtres cubes, bien qu'il soit plus élevé en moyenne. $\mathrm{Si}$, outre ce débit, on attribue un appont de 10 mètres cubes aux antres cours d'eau ce débit total serail de 330 mètres cubes. La puissance des cours d'eau est généralement basée sur le chiffre du débit moyen, et on aurait, de ce chef, une puissance moyenne de $330 \times 690=228000$ chevaux. Mais, outre que l'on ne voit guere quel usalge on pourrait faire d'une tolle puissance, d'ailleurs très inconstante, on a préféré liniter, pour le moment. la puissance de l'usine à l'emploi d'un maximum diennergic brute de 200 (100 chcvaux, pouvant foumir pratiquement une puissance maximum d'environ $150000 \mathrm{kw}$. en chiffie rond, à la ligne de transport.

Avec un clébit horaire variable, cette puissance de $150000 \mathrm{krw}$. pourrait etre transmise, en temps d'étige, pendant deux heures de la pointe, en conservant une puissance moyenne de $53000 \mathrm{~km}$. pendant le reste des 24 heures, toutes les variations de consommations désirables, méragées.

En temps de débit moyen, on a vu plus haut que cette puissance maxima te $150000 \mathrm{kw}$. serait alors disponible constammient. picmier mémoire (900 millimètres carrés pour chaque ligße). d'angmenter la puissance transportable. jusquat $150000 \mathrm{kw}$. el le rendement de la transmission jusqu'i près de 90 pour 100.

Mais les auteurs se réservent de recourit eventuellement it l'emploi de courants alternatifs triphasés ; car la nouvelle loi dor dishribulions d'énergirie ólectrique, qui rend désormais passible la pose ce supports plus encombrants sur les propriétés privées, pour une entreprisc d'utilité publique comme celle-ci, fail dispa"nitre en partie le motif principal qui forçait it empoloyer le courart continu, seul compatible avec une ofrupation des voies publiques far des eanalisations à très loaute tension (1).

Les calculs qui ont éti faits à ce sujet ont permis d'établir quarec une tension de 1:0000 rolts entre fils, pour laquelle des cmstructeurs penvent donner dies garanties núcessaires, on patr'rail, aved deux lignes suivant des parcours légerement differents, kransportey is Paris les $70000 \mathrm{kw}$. prévus pour l'utilisation dans la capitale, avec une chute de tension ne dépassant pas 20 pour 100 el avec une firequence de 25 perriodes.

Cetle solution permeltrait de n'avoir it la sortic de l'usine (qu'une tonsion relativement basse (10000 a 20000 volts), de transformel le coltanl pax des trinsformateurs clevadeurs it 1200 (n) volts et de supprimer at Paris toute usine de réception à machines

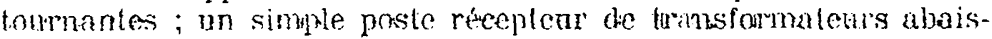
seurs ramenerait le comrant it 10000 on 15000 volts ; il serait uti-

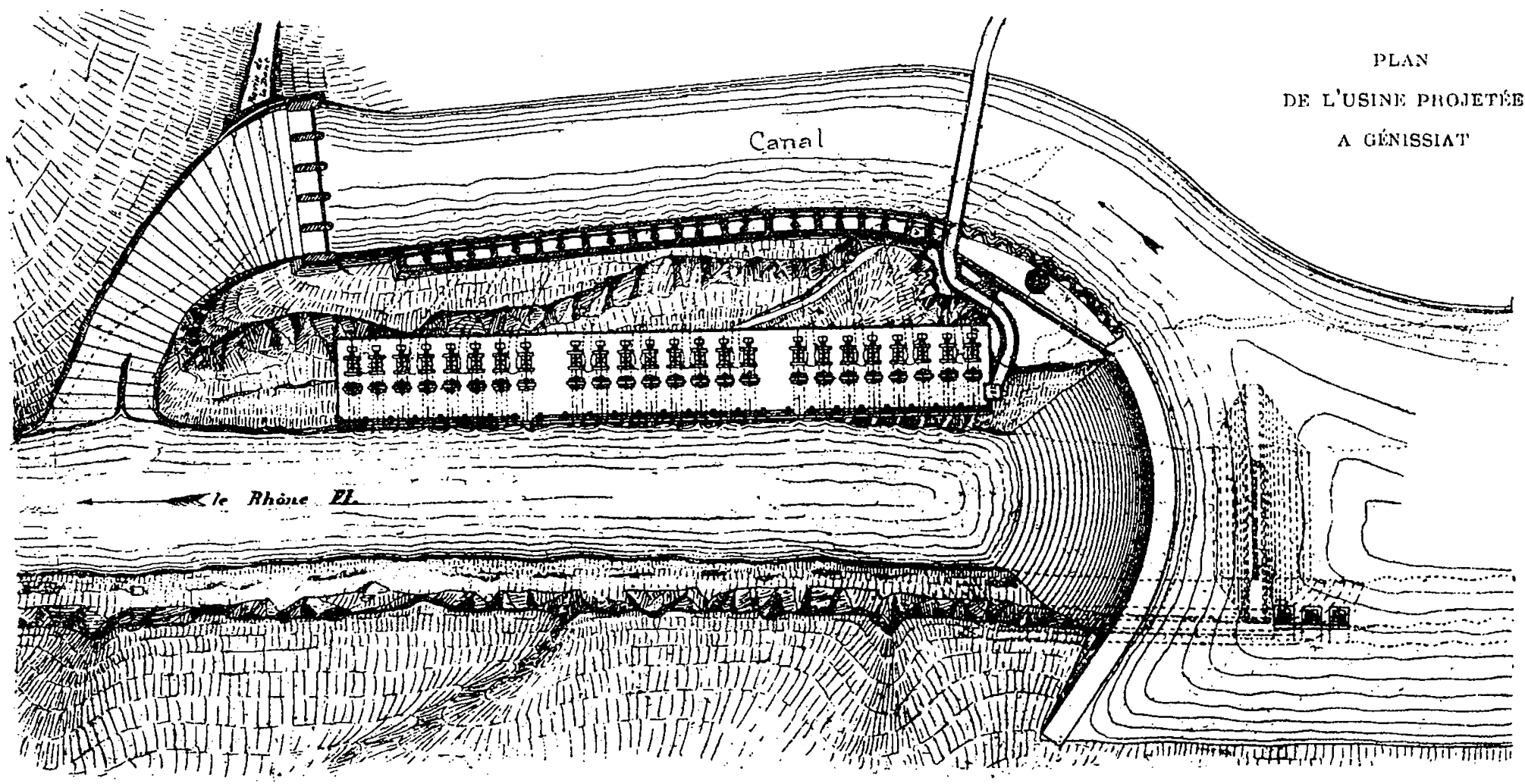

Dans ces condilions, on pourrait donc produire :

1. Pendant les 3 mois d'étjage, à raison de

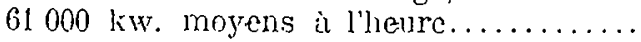

$2^{\circ}$ Pendant six mois d'eanı moyennes sans tenir comipte des cmucs.

$3^{\circ}$ Pendant 3 mois avec puissance intemne diaire entre les deux périolles.

132000000

650000000

228000000

Ensemble

1010000000

Soit un milliard de kw. heures par an, que l'ou n'utilisera cer tainemient pas en totalité.

Il n'y aura à prélever sur cette puissance que celle de 6000 chevaux environ nécessaire aux industries de la région de Bellegiturde.

Choix du courant à employer. - Dans le pl'écédent mémoire, on avait prévu I'emploi, pour le transport à Paris, de courant continu a 120 ono volts. Ce chiffre pourra être aujourd'hui dépassé, et porté sans inconvénient à 150000 ou 160000 volts, et peut-être même davantage, d'après les essais récents de Geneve. Cela permetitra, sans augmenter le courant normal, fixé à 1000 ampères ni par conséquent la section totale dos calbles indiquée dans le lisé alors concurmemment avec le courant fourni par les usines it vapeur et en parallide avec colles-ci. Dims ce cas, les alterna-

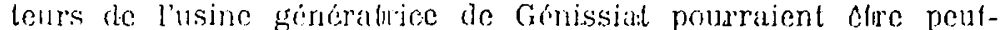
chre lomplaris par dos généralrices asynchrones, permetianl de faire toul le regrlage de tension allx usines a vapeur de Pands, suivant la solulion réaliséc récomment aver succes en Suisse pour les usines diz Binnenkanal.

Cependant juscu'ia nouvel ordre les auteurs du projel estiment loujom's que le courant ronlinu est celui qui donne le plus de: facilite pour l'installation des grandes lignes aŕriennes et de sé curite pour leur fonctionnement.

Usine genéralrice. - Comme on l'a dit plus haut, l'usine géné ratrice sera disposte lc long de la gorge du Rhone sur la rive droite, de facon a pouvoir sétendre librement en longueur. Cetle disposition sera analogue a celle de la belle usine toute récentr: des chutes du Niagara, de la "Ontario Falls Power Co ".

(1) De plus, n'itant plus astreints a suivre les bords des voies rubliques, les nombreux raccourcis que la loi du 17 juin 1906 autorise à eflectuer sur les propriétes privées, permetlent de reduire Je pareours total aux environs de 400 kilometres. Il nexcérlera ainsi que de $40 \mathrm{kiTo}$. niètres le transport de Colgate à Sin-Francisco. 
La production d'ćnergie sera assurce au moyen de groupes générateur's formes de puissantes lurbines de 10000 à 12000 chevaux, actionnant chacune un alternateur si l'on emploie les courants triphases, ou un groupe de deux ou trois machines a courant continu à haube tension, si l'on emploie le systeme Thury.

Toules ces unités généralrices scront dispjosées sur une seule rangee, au bas de la falaise, dans un local partie en béton armé, partie formese par la roche de la falaise en surplomb; le tout ólevé sur une banquelte rocheuse en bordure le long du fleuve, sur une larseur de 35 mètres environ. La longucur de l'usine seras d'environ 2\%5 mètres, et pour la commodité de l'exploitation clle sera divisee en 3 ou 4 groupes ayant chacun leur personnel de surveillanke. Les turbines du type Francis, par exemple, selont i deux roues, dont le diamètre ne dépassera pas 2 mètres, $11 i$ la vitesse 250 tours páa minute.

Ces tulbines doubles au nombre de 20 (et plus tard 21), seront sroupées par paires, chaque puire étant alimentée par une conduite forcéc de $2^{\mathrm{m}}, 70$ de diamètre, coudlée, et placée, comme on l'iz dit plus haut, a l'arrière des dynamos, au droit d'un puits incliné ; chaque conduite sera supportée convenablement et munie de deux joints de dilatation ; elle foumira leau à chaque turbine uru moyen d'unè vanne équilibrée, commandée par un servomoteur, et dic plus, la vanne Stoney correspondante à la descente sara commandéc électriquement au tableau de l'usine ; l'électricien de service pourra ainsi ouvrir et fermer à volonté toutes les canduites. L'eau sera amenée aux vannes des baches radiales des turbines par des culoltes en acier, et l'écoulement central se fera, de meme, par des culoltes aboulissant, par un coude de succion de 3 mètres de diamètre, à une conduite en béton construite dans des galeries, ou tranchées, ménagées dlans la banquetle rocheuse, el kébouchant obliquement dans le lit du Rhone, pour faciliter l'úcoulement. Ces conduites serront noyées en tout tompls, pour excrcer une action hydro-pneumatique sur les turbines, tout en pouvant clre isolves éventuellement par un batardean is l'aval.

On a choisi les turbines it axe horizontal, pour éviter les difficullés d'entretion des pirots, permettre l'emploi d'alternatcurs d'un meilleur rendement, et faciliter la surveillance et la visite de toules les porties, ainsi qu'éventuellement leur démontage.

Pour chaque roue, outre l'usage de la vanne, on modifiera l'inuroduction a l'aide de broches articulées commandées par un anneau commun à servo-moteur.

Premiere solution : Emploi du système Thury. - Les seules modicicalions par rapport au pusmier projet, en dehors de l'augmentition du nombre des unités motrices, seront les suivantes : la puissance unitaire des machines commandées par une meme turbine sera augmentée, et leur intensité de courant diminuée chaque turbine de 12000 chevaux commandera trois machines dynamos, debilant chacume 1000 amperes sous 2500 volts, au moyen de deux collecteurs. Chaque groupe générateur aura donc une puissance de $7500 \mathrm{kw}$. Les groupes seront a axe horizontal, afin de faciliter la visite des organes de l'isolement des dynamos, qui est la seule partie délioate d'une parreille installation; à ce sujet. le transport de Noutiers à Lyon a permis de démontrer quon peut réaliser un isolement parfait au moyen de blocs d'isolithe, sur lesquels on place les dynamos, avant de noyer toute la base dans un lit d'asphalte.

Tonts les conductour's soraient montès sur isolateurs a cloches, ou formés de cables armess à grand isolement, placés soit en sons-sol, soit sur consoles. Les sorties se feraient aux deux exlrèmites de l'usine, soit en cubles armés, soit en cables nus traversant deux grandes glaces épaisses enchassées dans une fenetre, puis s'élevant ensuite dorectement jusqu'au dessus de la falßise oủ ils rejoindraient la ligne aérienne. Pour la régulation, les turbires seraient divisées en 3 ou 4 groupes comme on l'a dit plus laut : pour chacun, les anneaux de réglage des vannages seront commandés par des tubes Mannesmann, montes sur frotlements à billes : chacun de ces tubes serait séparé en delux tronçons d'au plus 10 riètres de long, actionnés en leur milieu par un moteur électrique commandé par un régulateur.

Les régulateurs marcheront chacun pour leur comple, et pourront d'ailleurs etre rendus synchroniques dans leurs effets, par intercommunication convenable. On peut dailleurs laisser marcher sans réglage un ou deux des groupes d'unités travaillant a la charge la plus convenable, et laisser le réglage s'effectuer sur les autres groupes. L'asservissement sera réglé de farson que les régulateurs puissent toujours |rouver leurs positions d'équilibre, et que la charge puisse se répartir à peu près également. Pendant les heures de faible charge, on agira sur les régulateurs pour abaisser l'intensité ì 700 ampères, et rérluire la perte en ligne.

Les dynamos réceplrices seront du meme type, mais couplées individuellement chacune sur une dynamo ou un alternateur de puissance correspondante ; le réglage de vitesse sera obtenu par ciécalage des balais, comme dans les stations réceptrices de l.yon ; lexpérience a démontré que le fonctionnement est très satisfaisttht, grace a l'action très lapide du régulateur, qui évite des accidentis de collecteur en cas de court-circuit sur les lignes.

Deuxicme solution. Emploi des courants triphasés. - Dans le cas des courants triphasés, chaque turbine double commandera dincetement un allernateur multinolaire de $7500 \mathrm{kw}$., fournissant des courants triphasés de 25 périodes à 12000 volts; le diamètre tolal extierieur ilu stator sara d'environ $7^{\mathrm{m}}, 50$. L'encombrement de chacpe groupe de turbines et d'alternateurs sera d'environ 8 mètres dians le sens transversal, et 15 mètres dans la direction de l'arbse ; entre les unites voisines, on laissera un espacement de $2 \mathrm{~m}$; un espacoment plus grand sera ménagé entre les groupes de plusieurs unités reliés à un meme groupe de barres omnibus de lusine de distribution.

On aura 21 grancles turbines de12 000 chevaux environ, réglées ä vitesse constante par servo-motueurs hydrauliques, et 8 turbines de 600 chevaux adimentées par des conduites plus petites, pour l'entrainement des excilatrices, au nombre de deux pour chaque grompe de 8 alternateurs : l'une d'elles suffira pour l'excitation nolmate des inducteurs ; lautre servira de neserve. Ces excitatrices fourniront également à volonté du courant pour la manowure d'un pont roulant de 50 tonnes, et pour les circuits de comminnde des intenrupteurs à lıuile destinès ì couper les courants primaires ou serondaires. On pourra, du reste, joindre ì ces excitatrices une ballerie d'accumulateurs permetlant de commander ces appareils de manceuvre en tout temps.

Les courants de chaque générateur seront conduits aux barres de la slation de départ des lignes par des cables séparés, placés sur des supports en béton au-dessous du plancher ; ils passeront par des couteaux de connexion, par un disjoncteur à huile automatique, et par d'autres couteaux réunis a un cable triphasé armé ; ces apparcils seront supportés par des isolateurs dans une construction sprecialc en briques. Tous les cables seront logés dans plusieurs galeries soutenraines aboutissant à la station de départ des lignes aériennes. L'usine génératrice comprendra seulement un tableau pour l'excitation, et un autre pour l'éclairage et la force motrice nécessaires aux services de l'usine et des moteurs qui commandent les vannes; ces derniers moteurs seront munis de disjoncteurs limiteurs qui arreteront le mouvement des vannes dès que, dans l'une ou l'autre direction, elles auront atteint leur position extrême.

La station de départ de la ligne, qui sera installée sur le platea rocheux à une cortaine distance en arrière du canal de dérivation du Rhóne, conslituera le véritable poste de transformation et de distribution d'ou se fera le controle et la commande it distance des alternateurs, leur mise en charge et leur utilisation sur une ou l'autre cles lignes. Cette solution est celle de l'Ontario Power Company.

Chaque générateur, relié par un cable triphasé individuel, se trouvera commandé deepuis la station de départ comme sil y était installs ; une colonne placée dans la sabie de manoeuvre portera lous les appareils de mesure nécessaires, et les interrupteurs commandant a distance les interrupteurs automatiques de l'usine génératrice et ceux de la station de départ. Toules les barres principales scront on doubie, et les connexions par interrupteurs à huile seront disposées de manière à parmetlre d'alimenter par chaque génératrice, par l'intermédiaire de transformateurs, n'importe lanquelle des deux lisnnes de uransport ou directement la ligne de distribution lacale it 12000 volts. Chaque ligne de transport rocevra los courants à haute tension cl'une batlerie de 6 transformaleurs monophasés, soit deux par phase en parallèle. Ces transformateurs, qui elèveront la tension de 12000 à 120000 volts scront contenus chacun dans une bâche en acier fermée, munie seulement d'un tuyau de décharge de l'huile; le refroidissement sera fait par circulation d'eau. On ne coupera jamais la haute tension, mais seulement la basse tension. En cas d'accident a une ligne, on ouvrira immerdiatement les circuits des altornateurs travaillant on parallèle sur oelte ligne, qui sera déconnectée, et on les réunira immédiatement a l'autre ligne, qui sera surchargée jusqu'à la réparation de la première. En cas d'accident i un transformateur alimentant une ligne, on coupera de mène tout le couranit. sur les trois phases de cette ligne, en transfárant successivement tous les alternateurs sur l'autre ligne, puis 
on déconnectera le transformateur avarié pour le remplacer par un des transformateurs de réserve ; après quoi on rétablira les connexions primitives. Tous les connecteurs seront logés dans des cellules en briques et tous les circuits en cables seront isolés los uns des autres par des cloisons en brique.

La station de distribution, à deux étages, constituera un batiment presque aussi grand que l'usine de produclion du courant, et augmentera beaucoun les frais dinstallation a Génissiat. Mais l'amploi des courants triphasés permettra de réduire la station d'arrive à Paris à une station de transformation plus simple, et beaucoup moins chère a installer que la station réceptrice de la première solution. Il y aura seulement a prévoir des transformateurs statiques semblables à ceux de Génissiat, mais qui pourront avoir une puissance individuelle moindre; ils albaisseront les courants à 12000 volts environ, et on réglera par des inter. rupteurs à huile la répartition sur les différents goupres de feeders de distribution. Pour les usages industricls que les autcurs clu projet ont en vue, des varriations de voltage assez considérables sont sans inconvénient.

Lignes. - Dans la solution i courant continu, qui comporte cleux lignes, l'ume positive l'autre négative, leur constitution ne diffère de celle du premier projet que par la disposition meilleure des isolateurs, qui seront au nombre de 3, porlant chacun un cable de 300 millimèlnes carrés, et disposés cote à cole sur une traverse placée au sommet de pr-lones mélalliques plus forts. en profler pour espacer peut-ebre davantage les pylones en alignement droit.

Les lignes à courants triphasés seront aussi au nomlore de deux, mais travailleront en quantité ; et chacume d'elle pourra recevoir seule toute la charse pendant quelque temps, an prix d'une plus gratrde perte d'tnergie, si l'aulde est mise momentaniment hors de service.

Pour les conducteur's, il est prér'u trois cables par ligne, avec une section de 300 millimètres carrés, et une tension de 120000 volts.

Ces cables soront montés sur autant d'isolateurs de grand modele places aux trois sommets d'un triangle, sur de robusles tigies creuses évasées à la base et fixées at des traverses métal. liques.

Il n'y a aucune chance de rupture des conducteurs, qui auront du reste une fleche asse\% considrerable. Quant aux isolateurs, on est arrivé aux Elats-lnis, ef meme en liurope, it réaliser des qualités de poreelaine, et des fomes de cloches, assez satisfaisantes pour permettre d'affirmer sans crainte la possibjlité d'isoler une lifne mene triphasie it 120000 volts.

Distribution de lénergie dans laris. - Lo grand inléret du projet réskle daus la possibilile dalimenter à très has prix loutes les grandes entrentises de distribution d'endrgie, de comstruction mécanique, de transport en commun et tous les atclicrs importants qui ont besoin de force motrice electrique ; il ne sagrit pas,

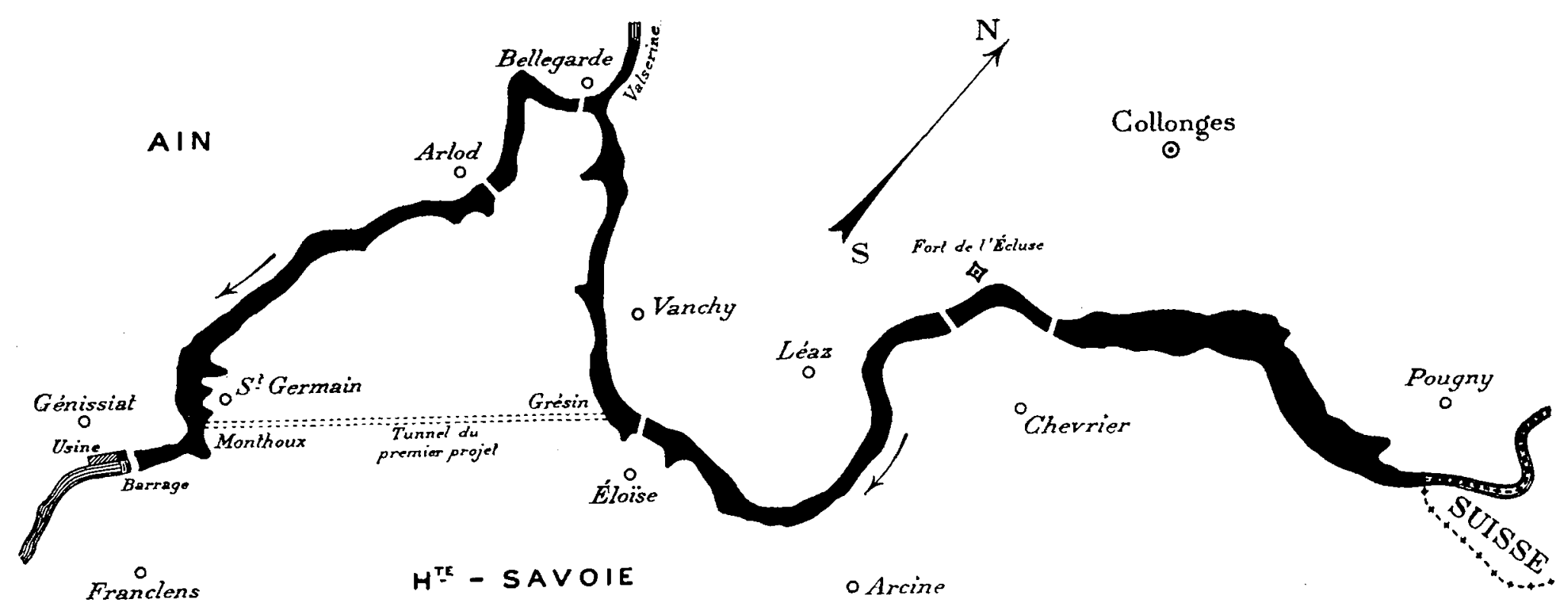

Les pylones seront espaces de 100 mètres en alignement droit ; les portées en courbe seront vieduites, ct les courbes seront réalisées d'une facon très progressive. Les pylones seront à grandes unailles et exćculess en fers profiles. Leur aspect fyénéral sera analogue a celui des pylones de la ligne il 80000 volts de la ligne de Lokport-Niagara-Ontairio.

La question de l'isolation est cntiorement rusolue pour les courants continus à haute tension. Les expériences exceutces à Genève, par N. Thury, ces temps demiers, jusqu'it 120000 volts, ont démontré que des isolateurs en bonme porcelaine résistent parfaitemenl, sans dimensions exagerées, it une dilférence de potenticl de 100000 volls par rapporl it la terre, el ne donnent lieu gu'à des pertes de courant insignifiantes, bien inférieures à ce que l'on avait estimé d’abord. Il a été fait par ailleurs d'inturessantes proposilions pour lemploi des cahles armés il placer dans le sol, et cetle solution pourra dtre prise en considération ulterieurement pour le courant continu sierie, si le prix nien est pas trop élevé.

Les lignes aćriennes à courant contimu pourrajent etre établies le long des routes nationales, sricc it leur faible encombrement. partout où on trouve hors des lieux habites des alignements droits assez longs pour éviter de trop fréquents supports d'angles ; elles réaliseraient d'ailleurs, en tout cas de longs raccour. ois a travers champs.

Les lignes a courants triphasés, dont les pylones sont plus encombrants, seraient installée en totalité sur les propriétes priviées; on pourra ainsi réduire le nombre minimum des angles, et par conséquent, de concurrencer les secteuls, ou leurs successeurs, dans la distribution de l'energie il hasse Iension pour leclainage et les petits moteurs d'appitrlement, mais bicn de fournir de l'énergic le plus direolement possible aux firos consommaleurs, par l"intermedjaire, soil d'une canalisaljon appartenant à 'a Ville de Paris, ou ì ses concessionmajes, soit à l'entropritse olle-mome, que la loi du 15 juin 1906 prermet de rendre independante de la coneession de liectairage electrique a Paris. Les auteurs du projet n'avainent pu tenir comple dans leur premier ménwire de celle facullé nouvel'e laissée frar la loj. Maj:; ils ont eru ulile ruvintenant denvisagar non seulement le cas d'une foumilure en bloc corrme dans le pretrier projel, majs aussit l'lypoutese d'une dishribution is haufe lension it tous les alronnes consonmant une force motrice de 25 chevaux et au-deli, all moven cỉun réseau de distribution speciw avec lension priinaire ì 12000 volts pour les grosses usines, et secondaire à 2010 volls pour les plus pelits consommaleurs it wablir dans tous les quartiers ou se presentent des groupes de consonumateurs le ce goure : le meme réseau fournirait l'énergic aux stalions transformatrices ou principales de tontes les entreprises qui en feraient li demande.

Cost ainsi, du reste, que l'on comprend actuellement en Anglctorre, non plus seulement dans les comtés, mais à Londres meme, la question des grandes distributions de la force motrice industiriellie it 2000 et 500 volts. C'est en partie la réduction do leur réseau de distribution au moyen de câbles de haute tension très économiques, (puisqu'ils n'exigent que de faibles poids do 
cuivre et de rares stations de transformation), gui explique les bas prix annoncés pour la venle de lénergie ; il suffira de prévoir daans ces condilions une dépense denvirou 250 francs par kw. distribué, soit pour $50000 \mathrm{~km}$, qu on installerait tout d'abord envirun 12,5 millions.

Les machines it vapeur existant actuellenent chez ceux qui

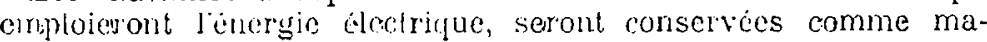
chines de secours. ce qui permettra de réduire à une faible puissunce lusinc à vipeur de secours nécessaire, si toutcfois l'une des grandes icentrales de la région nien tient pas lieu.

Darrs le cas d'une linne triphasce, la distribution a paris se fera dircelednent au sortir des transformateurs slatiques.

Au contraire, dans le cas du trinsport par courant continu, une trausformalion bal des groupes de machines loumantes, les unes recoplrices à courant continu, les autres generatrices a comranls alternatifs, est nécessaire.

Mais, commi on la dit clans lo premier projel, une partie des ruceptricess perurra servir plus avantageusement à commander diractement les machines génératrices à courant continu dans les sous slalions acluelles. Une moilie cnviron de lénergie serait transforne directement on courant triphase dlans ure usine construite a Ivry, et laubre, en courant continu, dans les sous stations do transforimalion ou dans des stations dautres entreprises. tolles quinsines géncatrices de tramways, de chemins the fer, ete.

Dans quelques quanticrs spécisine, faubours Saint-Antoine

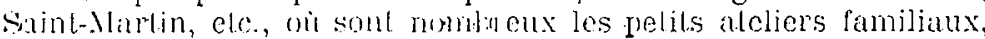
mn installerait 111 résmall secondaire spéciaul pour lil distribution de lat petile: force molrice it la tension de 500 volls; le reseau sero

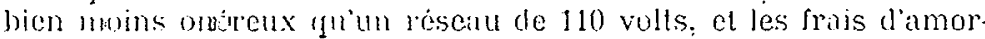

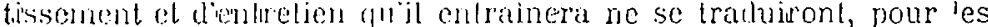
consommaleurs, gue par une faible augmentation du prix de vonle de lonergie, qui sera bien inférieur à coux qu'on pratique habihuellennt ; les auteurs du projet venlent qu't Paris l'onvier en chambre ait lénergie électrique a un prix au plus égral a reolui que patic le canut lyonnais.

Aucun des demandeurs ik-lucls n'est en étal de faire ressortir im prix de revienl aussi bas, par suite des charues énormes "quinurosent les canalisalions a basse lension el la consommation de charbon pirur la production de l'ènergie à lusine génératrice.

bistribulion en ronte. - Pour donner salisfaction aux drisirs des rérions traversies par les lignes, on distribuera en cours de roule quelrues miliens de kilowalls ; par exemple le déparlemont de l'yome a déja demaule de la force molnice pour son fulur riscau de tramways, afin de suphter aux insuffisances deau de la Curc. Milis ces fommilures ne diminueront pas sensimement les dismsitions dimergic pour paris, car toutes les chutes d'eau des payss de plaine ont leur ćtiaro en élé, alu lieu que celui du thone a licu en hiver.

Dépenses d'installation. - Les dépenses afférentes aux installations, dans lo nouvoau projet, sont peu dilferentes de celles du pnemier : on falit une économie asse\% notable par la suppression des deux fumnels, mais le barrage exire une dipense plus forte. Les exproprialions et le rétablissement des voies de communication alleignequt un chiltre plus élevé par suite de l'ovévalion de la relenue.

Dans ce qui suit, les depenses sont évaluées dans l'hypothèse de la retenue i la cote 332 . L'abaissement it la cote 327 entrairierait une exonomic de 600000 francs, sur les expropriations et dommages, mais une plus-value de 300000 franes sur les ouvrages de prise d'eau, fout en entrainant une réduction notable sur la puissamce disponible.

Les frais de l'installation de l'usine sont augmentés, par suite de l'nccroissement de la puissance de celle-ci, renforçé à $150000 \mathrm{kw}$, pour réaliser l'utilisation plus complèle des hautes eaux, sans compter linstallation ultericure d'aulres unites poul des emplois locaux intermitlents. La puissance moyenne transmise ¿ Paris est calculée d'après le débit de 210 mètres cubes; elle pourrait dtre augmentéc encore pendant les periodes de haules eaux, dans le cas de l'emploi du courant triphases au prix d'une chule de tension un peu plus forte dans la ligne. Par exces de précaution, on a continué à faire figurer dans les dépenses accessoires une somme ì valoir pour l"établissement evventuel d'un barrage régulateur it Pyrimont fou pour un aménagement du lac du Boururet), destiné à compenser les variations de débit introduites dans le régime du Rhone par l'emmaghasinement quoticlien pendant lectiage auquel on a fait allusion plus haut. Mais tout fajt prévoir, maintenant quion a. décidé la construction dusines a vaipeur de réserve a paris, qu'on préfèrera demander à l'usine hydraulique une puissance à peu près constante pendant la journée et dépendant seulement du débit moyen du Rhone à chaque saison ; dans ce cas, la retenue, loin d'augmenter les irrégularités du débit damemt, aurait plutôt pour efret de les anténuer.

La dorie d'exéculion des travaux pourra etre évaluée à 3 ans an maximum.

Ce délai est largenent suffisant si l'on se reporte au travail qu'a comparté le barrauge de l'urft et la distribution dans la région dAix-la-Chapelle. Pour avoir une puissance permanente de 3000 kilowatts, les Allemandls ont construit le plus haut barrage de l'Eurore; bien qu'avec $58 \mathrm{~m}$. 50 de hanteur il soit moins élevé que celui projeté ici, son cléveloppement de 226 mètres au sommet l'a rendu a'une exichation plus longne et aussi couteuse relalivement que le sera celui-ci dont le développenent n'est que de 140 mètres seulement an sommet. De plus, l'absence de route et do makerianx sur place avait fort compliqué lentreprise. Cependant, les travaux commencés on juillet 1901 élaient terminés en mai 1904, alor's mime quancune raison ne pressait leur achevement.

Il est inutile de dire rue le fait davoir des matérianx sur place of une gare a 150 mètres du chantier crée une situation exceptionmelle, el que les travaux autres que ceux du barrage, par leur fractionnement possible, malgir lour importance, ne peuvent etre une eause de relard pour l'entreprise.

\section{A. Travaux hydrauliques.}

I3arrage provisoire $\ldots \ldots \ldots \ldots \ldots \ldots \ldots$ Fr. 'Galeries de delivation of leur fermeture..... lir. Barrage définitif el accessoires............ lir. Camal damenée et prises dieau........... lir. Onvrige d'évacualion des crues............ Fr. Gurages pour le relevenent des voies de commu

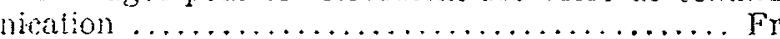

Expropriations .................... Fr

Travaux divers dans les gorges.............. Fr.

200.000

150.004

4.500 .00 !

1.500 .000

800.000

350.000

800.000

200.000

Somme it valoir $(25$ pour 100$) \ldots \ldots \ldots, \ldots \ldots$ Fr.

8.800 .000

2.200 .000

[hepense pour travaux hydrauliques. . Fr. 11.000 .000

\section{B. Usine generatrice}

Usine génératrice avec tous accessoires, en comprenant les turbines, dynamos-series, tableaux, canalisations électricues, etc., pour une puissance installée de 150.000 kilowalls ì raison de 60 franes par k"owtt ................................ Fr.

Ensemble. 9.000 .000

20.000 .000

La perte en ligne consentie ì pleine charge etant, estiméc à 10 pour 100 , la puissance nette de 150000 liiluwalts ne sera plus ia Paris que 135.000 kjlowalts.

\section{Lignes électriques}

I.es supports mélallicues espacés à 100 mètres seront au nombre de 9000 pour les deux lignes, d'un marcours d'environ 450 kilomètres chacune; le prix de ces supports en place, complètement équipés avec he pied bétonné, est estimé à 600 francs : soit pour les 9000 supporls....................... Fr.

A valoir pour angles, accessoires divers et imprévius ............................... Fr.

Pour 3 cables de $300 \mathrm{~m} / \mathrm{m}$ l de section par ligne, on aura, pour 900 kilomètres de l'ensemble, un poids de cuivre de $900000 \times 0,0003 \times 3 \times 8,8=7136800$ liloss, à $2 \mathrm{fr} .50$ le kilog., prix moyen................. Fr.

Ensemble pour les lignes électriques Fr.

24.000 .000

\section{Usine transformatrice de Paris}

Dipenses comprenant l'achat de $10000 \mathrm{mg}$ de terrains, construclions de batiments, transformateurs rolatifs pour 150000 kilowatis en comprenant les réserves, canalisations électriques de relations avec les réseaux primaires, diarrivée et de distribution, lableaux, etc., à raison de 70 francs par kilowatt ins-

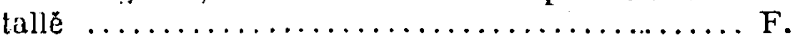

5.400 .000

i58.000 


\section{E. Récapitulation}

10 - Partie hydraulique et usine génératrice. Fr. 20.000.000

$2^{\circ}$. Lignes électriques ................. Fr. 24.000.000

$3^{\circ}$ - Usine transformatrice .............. Fr. 9.000 .000

A ces dépenses, il faut ajouter:

Somme a valoir pour une réserve d'eau ì créer à

Pyrimont ou au Bourget...................... Fr.

A valoir et intérêts intercalaires........... Fr.

Capital total.................. I.

Si lon estime la perte dans les transformateurs rotatifs et lignes de raccord a 15 pour 100 , on dispose d'une puissance utilisable de

$135000 \times 0,85=115000$ kilowatts.

D'où la dépense par kilowatt installé à Paris

$$
\frac{60000000}{115000}=522 \text { rancs }
$$

Et par kilowatt d'étiage :

$$
\begin{gathered}
\frac{60000000}{70000}=856 \text { francs } \\
\text { F. Dépenses fixes d'exploitalion }
\end{gathered}
$$

Les dépenses d'exploitation sont les suivantes : $1^{\circ}$ - 1 chief d'écluse et deux aides au barrage de Génissiat .............................. Fr. $2^{\circ}-1$ ingénieur et 2 chefs électriciens...... Fr. $3^{\circ}-15$ èlectriciens $\dot{a} 3.000$ francs......... Fr 4. -50 gardes-lignes a 1.200 francs......... Fr. $5^{\circ}$ - un ingénieur pour les lignes........... Fr. $6^{\circ}$ - Quatre surveillants supplémentaires à 1.500 francs $\ldots \ldots \ldots \ldots \ldots \ldots \ldots \ldots \ldots \ldots$ Fr. $7^{\circ}-12$ dectriciens ì lusine réceptrice, pour son siervice, $\dot{a} 3.000$ francs........................

$8^{\circ}-1$ ingénieur directeur et 2 chefs électriciens pour l'usine réceplrice ................... Fr.

$9^{\circ}-$ Bureaux dee la Direction............ Fr. $10^{\circ}$ - I.Ingénieur en chef du service central Fr. $11^{\circ}$ - Frais d'administration, de chemins de fer, téléphones, impols, assurance, redevances, elc. Fr.

$12^{\circ}$ - Pour grosses réparations, fournitures d'usinés $t$ t divers imprévus.................... Tr.

Total pour les frais dépendant de la production lir.

$13^{\circ}$ - Indemnité kilométrique de passage de la ligne, et prime de surveillance aux particuliers et aux communcs traversées, à raison de 0,125 le mètre li. néaire, soit environ....................Fr.

1.40 - Réserves à valoir pour travaux courants hydrauliques, réparations de lignes et divers... Fr.

$15^{\circ}$ - Intérêts et amortissement à 6 pour 100 en 50 ans sur la partie du capital relative aux ouvrages hydrauliques et bütiments en maçonnerie, soit de 15 millions environ...................... Fr.

$16^{\circ}$ - Intérêt et amort ssement en 50 ans sur les lignes électriques, soit de 6 pour 100 sur 2.1 mil-

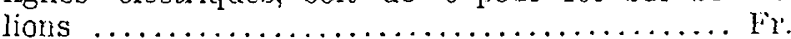

$17^{\circ}$ - Interest et amortissement en 20 ans en moyenne pour les orianes mécaniques et slectriques, c'est-ì-dine au taux de 9, 8 pour cent sur 12.500.00 Fr.

$18^{\circ}$ - Les frais supplémentaires d'entretien des supports et isolateurs de la lisne, en tenant compte des peintures et des plus frécuents remplacoments des isolateurs, soit......................... Fr.

$19^{\circ}$ - Intérét et amortisscment en 50 ans des sommes portées pour rachats, indemnités, interêts, somme à valoir, soit a 6 pour 100 sur $8.500 .000 \mathrm{fr}$. Fr.

A valoir pour imprévus................ Fr.
5.100

15.000

45.000

60.000

8.000

6.000

36.000

22.000

4.7 .000

15.000

500.000

240.600

1.000 .000

112.500

100.000

900.000

1.150 .000

1.225 .000

100.000

510.000

112.500

4.500 .000

Total des frais. constants............ Fr. En comprenant les frais de production. F.r.

1.000 .000

TOTAL GÉNÉRAL Fr.

5.500 .000 soit 9,18 pour 100 du capital engagé.
Ce rapport est satisfaisant, cu égard à la longue durée de la concession et i la grande puissance installée d'un seul coup.

lin répartissant les frais variables sur un minimum de 200000000 de lilowatts-heure, on obtient ainsi la formule binome du prix du hlowalt-heure en fonction du nombre de kilwwatts-heures venclus, $m$.

$$
p=\frac{4500000}{m}+\frac{1000000}{200000000}=\frac{4500000}{m} ; 0 \text { ir. } 005
$$

ou en fonction du nombe $n$ d'heures dutilisation de la puissance des abonnés riguliers a Paris, qu'on peut estimer a ro 000 kilowatts, conme dans le premier mémoire, et pour les memes raisons (sans parler des fournitures facultatives intermittentes).

$$
p=\frac{4500000}{70000 n}+0 \mathrm{fr} .005=\frac{(34,3}{n}+0 \mathrm{fr} .005
$$

En donnant à la durée d'utilisation annuelle $n$ de chaque kilowatt

\begin{tabular}{|c|c|c|c|c|c|c|}
\hline $\begin{array}{l}\text { Durie de fonction- } \\
\text { nement innuel di } \\
\text { charatue kilow. - iustalle. }\end{array}$ & \multicolumn{2}{|c|}{ 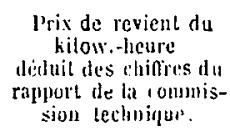 } & \multicolumn{2}{|c|}{ 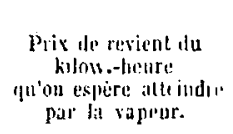 } & \multicolumn{2}{|c|}{ 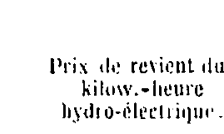 } \\
\hline 1.000 heules & 16,5 & entimes & 10,8 & nlimes & 6,9 & entimes \\
\hline $1.200-$ & 15,1 & - & 9,8 & $\ldots$ & 0,0 & - \\
\hline 1.500 & 14,0 & - & 8,7 & - & 4,8 & - \\
\hline 2.000 & 13,0 & - & 7,6 & - & 3,7 & - \\
\hline $2 . ?, 00$ & 12,5 & - & 7,0 & - & 3,1 & - \\
\hline 2.800 & 12,1 & - & 6,7 & $\ldots$ & 2,8 & - \\
\hline 3.000 & 12,0 & - & 6,6 & - & 2,6 & - \\
\hline 6.000 & 11,0 & - & $5, \overline{5}$ & - & 1,6 & - \\
\hline
\end{tabular}
installé des valeurs successives, le prix de revicnt du kilowaltheure varie dans les condlitions suivantes, qu'il convient de mettre en regard de ceux qu'on espère obtenir dans les usines it vapemir installées dans les conditions les plus favorables:

Il convient d'ajouter que les frais fixes de 0,005 doivent s'abilisser fortement avec une consommation abonklante et avec 3.000 heures d'utilisation des unités, ils doivent certainement etre it peine de 0,0025, ce qui abaisserail le prix dı kilowatt-lıure ver's

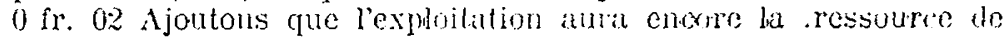
la vente de ba foree resibuaire it des industries lowales.

Bien plus, at la fin de la période d'anoorlissement, dtant domice lublilisation horaire ammelle des uniles installeses, la depense ne? se ratrportera plus gu'a l'entretien el a des frais fixes moindres de 0,2 centimes ; d'oì le kilowath-hente varieril en prix de revient entre 1 centime et 0,5 centimes; lanklis gue par la vapeur il auria allgmenté aree les prix du charbon.

Emploi du courant triphases. - Dans la variante it couraul triphasé, les dipenses tolales seraient it peu pres les monnes ct. se réparticaient autrement. Lusine receptrice serait beauenul simplifiée par l'emploi de simples transformateurs slatiques, mais l'économie réalisée de ee chef serail compensée par la dépense

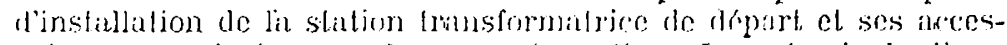
soires, fule n'exige pas le comrant continu. Le prix de la ligne serail sensiblement lo metme dans les deux cas, mais la puissance nette trinsmise it Paris serait miljorće de 10 pour 100 environ qu'on economisera sur la transformiation. Lin effet, les transformateurs statiques ne consommeront pas pius de 3 pour 100, pour l'élévation et l'abaissement de corssion réninis, alors qu'un groupe moleur-sóntrateur formé dione réceptrice et d'un. alternateur aurait difficilement un rendement supérieur it 12 ou 13 poul lok). On pouria done obtenir au sortir de lusine une puissance supirieure it 125000 kilow. all lieu de 115000 , co qui compensernit la perte phus álevere dans la lians (1).

(1) Les auteurs din projet font remarquer que lon pourrail prut itre oljecter atux évaluations ci dessus quo l'on a admis des tensions dec triques supírienres ì celles acturllement en usage, at que lo prix de la ligne serait bien plus álevé si on ne pouvait réaliser ces prévisions: Mais il est facile de voir, pall exemple. rans le cas drs connants triphasćs. que jemploi d’unc tension de 80.000 volts, actuellement réaliséc phases. que lemploi dunc tension de 80.000 volts, actuellement realised - dans plusieurs transmissions anx bata-Unis, au liev de 120000 présus,
n'entrainerait quane majoration de 90 millions sur le prix des lienes n'entrainerait quine majoration de 20 millions sur le prix des lignes
ilectrifues, majoration qui ne compromet pas sensiblement le resultat espéré.

D'ailleurs une tension de 150.000 volts est déja adoptée en principe pour Je transport d'énergie des chutes de Victoria à Johamnesburg, et les experts techniques américains la considerent cornme déji pratirque: ment rénlisable. 
Cas d'une distribution de force motrice. - Si notre entreprise obtient, comme clle le desire, le droil de poser des canalisations spéciales pour une distribution de force motrice (comme celle de Lijon et celle projetée pour Londres), on peut estimer comme il suit les dépenses supplementaires, en supposant 70000 kilowmoyens vendus aux abonnés.

Frais detablissement. -.. Lignes is 10000 et 2000 volts pour 50000 kilow. vendus, à 300 francs......

Transformateurs et lignes sccondaires it 500 volts pour 20000 kilow. it 500 francs.............. 10.000.000 "

Total....... $25.000 .000 \mathrm{fr}$.

ce qui donne une moyenne de 350 francs par kilow, vendu.

p'rix de venle par lillowalt-an. - Les frais d'intérèt et d'amortissement et d'exploitation $=10 \%$ de 350 francs $=35$ francs.

Ces charges fixes seronl ainsi portées de 64 francs (chiffre donne plus haut) i 100 frantes en chiffe rond. Les charges mobiles scraient augmentées d'environ 1 centime.

On rentalquera que, pour abaisser le prix de la force motrice chez louvrier, on répartira les frais de distribution sur tous les consommaleurs.

En chiffre rond, le prix de vente chez l'abonné de force motrice devient ainsi, en francs, par kilowatt-al! :

$$
P=100+0,015 n
$$

$n$ étant le nombre dheures. Il on résulte pour le kilowattan $P$ et lo kilowaltheure $p$ les prix suivants :

$\Lambda$ vec 3000 kilow. par an, $P=1$ íj fl. soit 105 fr. le cheval ; et $p=0,018$ environ.

Avec (jolo kilow. par an, $P=190$ fr. soit $13 \pi$ fr. le cheval ; et $p=0,03$ environ.

Aures atantages grincraux de la nouvelle solution. - Les avantages de la nouvclle solution sont considerables, au point de vue gênéral.

$1^{\circ}$ Lat durée el les frais d'installation se trouveront diminués par la suppression des longs tumnels prévit; dans le premier projet : on evitera ainsi tout chomage partiel pouvant résulter de réparálions à l'un des tunnels.

20 Lílulisalion de la puissance du Rlone alleindra son maximurn ; on réalisera ainsi le but auguel dojt tendre touls administrution soncieuse des intercts de la collectivilie, it savoir tirer pour celle collectivite le maximum darantanes possibles des richesses naturelles : aucum des projels antérieurement concus pour l'ulilisation des caux du Rhone ne présente ces memes avantages; colui-ci est réellenent un projet d'utilité publique maxima.

Tous les excedents de force disponijle penxlant la période des hantes eatux pourront etre ainsi employes, soit it fournir une plus grande qumbite deneroie it bom marche is Paris, ou dans les villes taversces par le projet, soit it almenter cerlaines installatimis locales, ou ì crécr de nouvelles applicalions de lélactrochimic. Il est inléressant de remariuer que les haules caux du Rhone correspontiont aux itiages des rivieres des régions traversies, et guon pourrait satisfaire aux besoins d'énerurie de ces régrions au moment oul elles on ont le plus ljesoin, sans gener le transport sur Paris.

Les frais d'exploitation de l'usine unique seront très minimes en comparaison de ceux des trois usines qu'exigerait la division du Rhone en relenues partielles. Le prix et le rendement des turbines et dynamos est réduit d'une façon importante par l'emploi d'une haute chule unique au lieu de plusieurs. Les dépenses d'installations seront faites seulement au fur et it mesure que le placement de l'énergie correspondante sera assurée, afin de réduire au minimum le capital improductif.

$3^{\circ}$ Il sera mis fin allx érosions du lit du Rhone entre la frontière suisse et Génissiat ; une voie d'eau naviusable sera créée entre ces deux points : pent-etre pourrait-elle meme plus tard etre utilisće, si l'on créait à Gémissiat un ascenseur à bateaux, et il deviendrait alors facile didablir la communication par eau entre Lyon et Geneve, et de réaliser ainsi un reve concu il y a plus d'un siècle par Céard, l'auteur célèbre de la route du Simplon; on aplanira ainsi la plus grande difficulté de la mise en relation de la Suisse avec Iyyon et la Méditerranée.

$4^{\circ}$ Enfin, l'utilisation du lac du Bourget, comme réservoir compensateur, permettrait non seulement de rendre insensibles les variations du régime du Rhone provoquées par les valliations diurnes du fonctionnement de l'usine, mais encore d'ameliorer la navigabilité du Rhône à Lyon.

\section{SYSTÈME DE SIGNES CONVENTIONNELS} pour la représentation des Appareils et Installations électriques (1)

Dans le courant de 1905, la sixième Section de votre Comité a été saisie, par notre collègue $M$. Vedoveli, d'une proposition tendant à mettre à l'étude un système de signes conventionnels pour la représentation des appareils et installations électriques.

M. Vedovelli soumettait, en même temps qu'une liste d'appareils très détaillée, un grand nombre de signes s'y rapportant, susceptibles d'un emploi courant et sur lesquels pouvait s'exercer la discussion et le choix de la Société des Electriciens. Ce tableau préparatoire constituait une base de travail pour un projet d'un caractère plus général, dont l'étude fut confiée à une sous-commission composée de MM. Aliamet, Boistel, Hospitalier et Pérot, et dont j'eus le plaisir de faire partie.

Les propositions de cette sous-commission furent ensuite discutées en détail sous la direction de notre sympathique et éminent Président de la sixième Section, M. Guillaume, au cours des séances régulières de cette Section. La question, quoique d'un intérêt purement professionnel, mérite cependant, croyons-nous, un moment d'attention.

J'ai l'honneur de vous rapporter aujourd'hui, à ce sujet, les propositions de la sixième Section.

Jusqu'ici, à notre connaissance, et l'on peut s'en convaincre par l'examen des publications techniques périodiques, aide-mémoire, etc., il n'a pas été préconisé de méthode pour le choix et l'application des signes conventionnels relatifs aux installations électriques.

La représentation des schémas, au contraire, n'a eu comme règles que le goût ou l'habitude propre à chaque ingénieur ou maison de construction ; la pratique a cependant rendu classiques quelques signes. La sixième Section a eu grand soin de les incorporer dans les tableaux définitifs dont elle préconise l'emploi.

C'est pour remédier à l'état de choses que nous venons d'indiquer que la sixième Section a cru bon de poursuivre l'établissement d'un systeme de signes conventionnels; disons de suite qu'elle s'est limitée aux appareils et intallations de transport, de distribution et d'utilisation d'énergie électrique, laissant de côté, au moins pour le moment, les installations de télégraphie et de téléphonie.

Il a semblé également à la sixième Section que l'uniformisation des signes conventionnels était appelée à rendre des services incontestables.

En effet, les schémas, vu le développement des installations, deviennent de jour en jour plus compliqués, et la diversité des signes choisis par les constructeurs et les exploi. tants ne peut que rendre la lecture et la compréhension plus pénibles.

Une écriture symbolique, simple, claire, facile à retenir, comportant un minimum de signes combinables entre eux, devait être relativement facile à établir pour répondreà tous les besoins; les règles à suivre devaient être également simples et d'une application très élastique. Tels sont les principes qui ont guidé la sixième Section.

Veuillez bien retenir que l'idée d'une réglementation de ce genre n'est pas nouveile.

(1) Communication de E.-J. Brunswik à la Société Internationale des Electriciens, séance du 6 février 1907. 